\title{
Recrystallization of Water in Non-water-soluble (Meth)Acrylate Polymers Is Not Rare and Is Not Devitrification
}

\author{
Makoto Gemmei-Ide*, Atsushi Ohya, and Hiromi Kitano* \\ Department of Environmental Applied Chemistry, Graduate School of Science and Engineering, \\ University of Toyama, 3190 Gofuku, Toyama 930-8555, Japan
}

(M.G.-I.) E-mail: ide@eng.u-toyama.ac.jp; Tel: +81-76-445-6870; Fax: +81-76-445-6703.

(H.K.) E-mail: kitano@eng.u-toyama.ac.jp; Tel: +81-76-445-6868; Fax: +81-76-445-6703.

\begin{abstract}
Change in the state of water sorbed into four kinds of non-water soluble poly(meth)acrylates with low water content by temperature $(T)$ perturbation was examined on the basis of $T$ variable mid-infrared (MIR) spectroscopy. Many studies using differential scanning calorimetry suggested that there was no change in the state. $T$ dependence of their MIR spectra, however, clearly demonstrated various changes in the state. Furthermore, recrystallization, which was crystallization during heating, was observed in all four polymers. Recrystallization observed in this study was not devitrification, which was the change in the state from glassy water to crystalline water, but vapor deposition during heating (vapor re-deposition). There were only two reports about recrystallization of water in a non-water soluble polymer before this report; therefore, it might be considered to be a rare phenomenon. However, as demonstrated in this study, it is not a rare phenomenon. Recrystallization (vapor re-deposition) of water in the polymer matrices is related to a balance between flexibility and strength of the electrostatic interaction sites of polymer matrices but might not be related to the biocompatibility of polymers.
\end{abstract}

Keywords: Change in the State/ Sorbed Water / Vapor Re-deposition / Biocompatibility

\section{Introduction}

Various synthetic polymers have been used as base or coating materials for biomedical devices such as contact lenses, vascular grafts, heart-lung machines, and artificial kidneys. The ability of surfaces to prevent protein adsorption and cell adhesion, that is, biocompatibility, is required for such applications of synthetic polymers. The biocompatibility is known to depend on charge, wettability, free energy, roughness, and stiffness of the polymer surface, but a universal explanation for the origin of it has not yet been obtained. ${ }^{1-5}$ Polymers and biological components have infinite variations in their chemical structures. Water, on the other hand, is the only common substance when biocompatibility is investigated; therefore, the physicochemical property of water (water structure) is expected to play a crucial role in biocompatibility.

Results of some studies on the relation of biocompatibility to water structure in aqueous polymer solutions ${ }^{6-9}$ and non-water soluble polymers ${ }^{10-22}$ have been reported. Among them, the water structure in poly(2-methoxyethyl acrylate) (pMEA), which is a non-water soluble polymer and a polymer with high blood compatibility, has been examined by differential scanning calorimetry (DSC), ${ }^{10-16}$ X-ray diffraction spectroscopy, ${ }^{17}$ nuclear magnetic resonance spectroscopy ${ }^{18}$ and mid-infrared (MIR) spectroscopy. ${ }^{19-22}$ On the basis of results of DSC analysis, it was proposed that biocompatible polymers have "intermediate water" (cold-crystallizable water or recrystallized water or freezing bound water). ${ }^{10-16}$ "Intermediate water" was defined as water that is frozen (crystallized) only during heating. In the general definition in DSC, however, "intermediate water" is water that is frozen or molten at a lower temperature $(T)$ than the thermodynamic melting $T\left(T_{\mathrm{m}}\right)$ of water, $273 \mathrm{~K},{ }^{23}$ regardless of the direction of $T$ change. To avoid confusion in this report, the term "recrystallized water" is used.

There have been some reports based on DSC analysis about recrystallized water in aqueous polymer solutions. ${ }^{24-28}$ Recrystallization of water is generally considered to be devitrification that glassy water crystallizes during heating. In the case of non-water soluble polymers, on the other hand, there have been only two cases of pMEA $^{10}$ and poly(tetrahydrofurfuryl acrylate) (pTHFA), ${ }^{14}$ as far we know. pTHFA is also a polymer with high blood compatibility. It was thought that recrystallized water in the two polymers also originated from glassy water and was in a condensed state, which had a special structure to avoid protein adsorption, at physiological $T .^{10-16}$

It is not clear whether the recrystallized water is a necessary and sufficient condition for biocompatibility, and the aim of this study was not to verify that recrystallized water induces biocompatibility. Clarification of the correlation of water structure to the biocompatibility of polymers was also not the aim. The aim of this study was to determine whether recrystallization of water in non-water soluble polymers is physicochemically the same as that in aqueous polymer solutions, that is, devitrification. This is because if the consideration about pMEA and pTHFA is correct, it 
means that the formation of glassy water is achieved by a very slow cooling of $2.5 \mathrm{~K} \cdot \mathrm{min}^{-1}$. Under ambient pressure, the preparation of glassy water generally requires rapid cooling (quenching) of the system, and there have, therefore, been no reports of preparation of glassy water without quenching. In the present study, four kinds of poly(meth)acrylates having recrystallized water were newly discovered spectroscopically and, on the basis of investigation of change in the state of the sorbed water by $T$ variable MIR spectroscopy, it was examined whether recrystallization of water in non-water soluble polymers is devitrification or not. The four poly(meth)acrylates examined were poly(methly acrylate) (pMA), poly (n-butyl methacrylate) (pBMA), poly(ethoxyethyl acrylate) (pEEA), and poly(ethoxyethy methacrylate) (pEEMA). The differences in the chemical structure are as follows: (1) the two latters (pEEA and pEEMA) have ether group in the side chain as the same as pMEA, whereas the two formers not; (2) pBMA and pEEMA have $\alpha$-methyl group in the main chain, whereas pMA and pEEA not. There have been no reports that the four polymers are the biocompatible materials.

Before beginning Results and Discussion, mention should be made here of several terms used in this report. The terms "condensation", "vaporization", "vapor deposition", "sublimation" and "vapor re-deposition" were used for expressing the change in the state of the sorbed water in the polymer matrix. From the point of view of physical chemistry, these five terms would not be suitable because monomeric water in the polymer matrix should not be in a gas state but associated with polymer chains via electrostatic interaction. However, if the polymer chains are omitted, the change in the state from/to monomeric to/from condensed state might be able to be regarded as the above expressions. The term "crystallization" is generally used for the change from liquid water to crystalline water. In this report, however, it was used for the formation of ice regardless of its process. Instead of this, the term "freezing" was used for the change from liquid water to ice.

\section{Experiments}

Poly(methyl acrylate) (pMA), poly(n-butyl methacrylate) (pBMA), poly(2-ethoxyethyl acrylate) (pEEA), and poly(2-ethoxyethyl methacrylate) (pEEMA) were prepared by a conventional free radical polymerization of corresponding monomers in toluene at $350 \mathrm{~K}$ using 2,2'-azobisisobutyronitrile as a thermal initiator. Their weight-averaged molecular weights $\left(M_{\mathrm{w}}\right)$ and polydispersity indices $\left(M_{\mathrm{w}} / M_{\mathrm{n}}\right)$ determined by gel permeation chromatography are given in Table 1 . Measurement of $T$ dependence of the MIR spectrum of sorbed water has been described. ${ }^{29,30}$ Briefly, the sample, which was prepared as a film on a sapphire substrate of $13 \mathrm{~mm}$ in diameter by a solvent-cast method, was hydrated by exposing it to humid air with $88 \%$ relative humidity at $310 \mathrm{~K}$ for 10 days. The water content of hydrated samples in weight percent was determined by the Karl Fischer method (Table 1). The sample was set in a homemade sample-holder and then tightly sealed with indium so that the water content was constant during the measurement. MIR spectra of the hydrated and dried samples were recorded on a Spectrum One NTS (Perkin-Elmer) with a resolution of $4 \mathrm{~cm}^{-1}$ and a scanning time of $20 \mathrm{sec}$ in a temperature (T) range from $298 \mathrm{~K}$ to 170 $\mathrm{K}$. Cooling and heating rates were $0.5 \mathrm{~K} \cdot \mathrm{min}^{-1}$. The temperature of the sample $(T)$ was controlled by an Optistat-DN cryostat (Oxford) connected to a model ITC601 $T$ controller. The spectra of the sorbed water were obtained by subtraction between the spectra of the hydrated and dried samples at the same $T$.

\section{Results}

Spectrum at 298 K (Figure 1). MIR spectra of water sorbed into the four polymers and the spectrum of pure water at $298 \mathrm{~K}$ are given in Figure 1. Pure water has a broad main peak centered at $\sim 3400 \mathrm{~cm}^{-1}$ and two shoulders at 3630 and $3280 \mathrm{~cm}^{-1}$ in its MIR spectrum. The higher frequency shoulder is attributed to three-coordinate asymmetrically hydrogen $(\mathrm{H})$-bonded water molecules. ${ }^{31,32}$ The lower frequency shoulder is generally attributed to the symmetric $\mathrm{OH}$ stretching mode $\left(\mathrm{v}_{\mathrm{OH}}\right)$ of tetrahedrally coordinated water molecules (strongly H-bonded water molecules) and the main peak is attributed to the $v_{\mathrm{OH}}$ of asymmetrically H-bonded water molecules. ${ }^{31,32}$ On the other hand, the spectra of water sorbed into the four kinds of polymers are very different from the spectrum of pure water, suggesting that most of the sorbed water is associated with electrostatic interaction sites of the polymers and is not in liquid state. In the higher frequency region over $3500 \mathrm{~cm}^{-1}$, two bands at $\sim 3615$ and $\sim 3540 \mathrm{~cm}^{-1}$ and an obscured shoulder at $\sim 3690 \mathrm{~cm}^{-1}$ are observed.

Recently, Iwamoto ${ }^{33}$ and Iwamoto et al. ${ }^{34}$ reported MIR spectra of 1:1 and 1:2 complexes between water molecule and di- $n$-butyl ether, 3-decanone, or methyl decanoate in $n$-heptane and proposed band assignments for the three components mentioned above. Band assignments were made according to them in this report. Water molecules in the polymer matrix should be bonded to the electrostatic interaction sites with both of their $\mathrm{H}$ atoms or with one of their $\mathrm{H}$ atoms. The former and the latter are denoted as "bridging water" and "dangling water", respectively. The high frequency shoulder at $\sim 3690 \mathrm{~cm}^{-1}$ is attributed to the stretching mode of the non-H-bonded OH group of dangling water. The high frequency band at $\sim 3615 \mathrm{~cm}^{-1}$ is attributed to the asymmetric $\mathrm{OH}$ stretching mode of the bridging water. The low frequency band at $\sim 3540 \mathrm{~cm}^{-1}$ has two origins: (1) stretching mode of the H-bonded OH group of dangling water and (2) symmetric $\mathrm{OH}$ stretching mode of bridging water. The intensive band from bridging water and the weak band from dangling water indicate that most of the sorbed water is bridging water.

In the case of pMA, pEEA and pEEMA, two small shoulders were observed at $\sim 3450$ and $\sim 3250 \mathrm{~cm}^{-1}$. As stated 
above, the two bands in this low frequency region are generally considered to be weakly and strongly H-bonded water molecules (between water molecules). At present, it is unclear whether this water is liquid water or a small water cluster. The amount of this water, however, should be small because the absorption coefficient is strongly enhanced by fully H-bonding formation. ${ }^{35}$

After all, most of the sorbed water in the four polymers was found to be the bridging water. The higher frequency band at $\sim 3615 \mathrm{~cm}^{-1}$ rising from the bridging water is well isolated from the spectrum of pure water. This band intensity is used as an indicator of the change in the amount of the bridging water, that is, the monomolecular water isolated in the polymer matrix.

Spectral Changes by $\boldsymbol{T}$. Figures 2, 3 and 4 show $T$ dependence of the MIR spectrum of pure water and the spectra of water sorbed into pBMA and pEEMA, respectively. The results for pMA and pEEA are given in Figures S1 and S2, respectively, in Supporting Information (SI) because they are similar to those of pBMA or pEEMA. The right and left panels for each figure show the results at cooling and heating, respectively. Panel (a) shows the original spectra selected at every $\sim 10 \mathrm{~K}$ from $298 \mathrm{~K}$ to $170 \mathrm{~K}$. Panel (b) shows $T$ dependence of a peak absorbance assigned to the bridging water (A $x x x x$, top) and that of a peak absorbance at $\sim 3280 \mathrm{~cm}^{-1}$ (A3280, bottom). Arabic number " $x x x x$ " of Axxxx corresponds to a peak position of the bridging water at $298 \mathrm{~K}$. In the text, "Axxxx" is denoted as "A $\mathrm{A}_{\mathrm{MONO}}$ ". Vertical lines in panel (b) indicate maximal, minimal, or bending points of $\mathrm{A}_{\mathrm{MONO}}$ or A3280. Arabic numbers on the vertical line are their $T$ 's. $C X$ or $H X$ (X: Arabic number) is a numbering for $T$ range divided by the lines. Panel (c) shows the difference spectra between two original spectra at both side $T$ 's in each $T$ range. $C X$ or $H X$ on the left top side of each spectrum corresponds to that in panel (b). If jump-up/-down of A3280 was observed, the numbering in panel (b) was placed on the vertical line and the difference spectrum in panel (c) was calculated from the two original spectra at $\pm 1 \mathrm{~K}$ of the jump point.

The values of $\mathrm{A} 3280$ and $\mathrm{A}_{\mathrm{MONO}}$ were the presented peak intensity of the IR spectra without a band resolution (for example, the Gaussian deconvolution). The component assigned to the bridging water was also overlapped with the other component assigned to the dangling water: therefore, the change in the value of $\mathrm{A}_{\mathrm{MONO}}$ was not equal to the change in the amount of the bridging water. However, the value of $\mathrm{A}_{\mathrm{MONO}}$ should be used as an indicator of the change in the amount of the monomolecular water, because both the bridging water and the dangling water are the monomolecular water isolated in the polymer matrix and the IR component of the bridging water was separated from that of liquid water. On the other hand, the value of A3280 might have a double origin (the small water cluster and liquid water (ice)): therefore, the value of A3280 cannot directly give the exact amount of liquid water (ice). However, as seen in the case of pure water (Figure 2), the ice formation can be decided from the discontinuous change in the value of A3280 and the spectral profile.

A. Pure Water (Figure 2). By cooling, MIR spectra of pure water with a very broad feature above $251 \mathrm{~K}$ changed promptly to those with a sharp band at $\sim 3280 \mathrm{~cm}^{-1}$ (panel (a)). Oppositely, by heating, the sharp band returned to the broad one above $273 \mathrm{~K}$. The two changes are freezing and melting, respectively. The sharp peak at $\sim 3280 \mathrm{~cm}^{-1}$ in the lower $T$ side characterized ice $I_{\mathrm{h}}$, but before freezing (after melting), only a shoulder was observed. If there was no clear peak at $\sim 3280 \mathrm{~cm}^{-1}$, the value of A3280 was adapted to an absorbance fixed at $3280 \mathrm{~cm}^{-1}$. This rule was also applied to the case of sorbed water. Jump-up and -down of A3280 were observed at $251 \mathrm{~K}$ and $273 \mathrm{~K}$ in cooling and heating, respectively (panel (b)), indicating freezing and melting of water, respectively. The lower freezing $T$ than $T_{\mathrm{m}}$ of water is merely supercooling.

The two difference spectra (panel (c)) on diagonally opposite sides have a mirror-image relation. In the $T$ ranges of $C 1$ and $H 3$, water is in the liquid state. The negative/positive $\left(\sim 3600 / \sim 3450 \mathrm{~cm}^{-1}\right)$ bands observed during cooling indicate that water molecules make a more associated form by $T$ reduction. ${ }^{35-37}$ At the liquid-solid transition $(C 2$ and $H 2$ ), a clear peak at $\sim 3250 \mathrm{~cm}^{-1}$ and two shoulders at $\sim 3400$ and $\sim 3150 \mathrm{~cm}^{-1}$ and an asymmetric band at $\sim 3500 \mathrm{~cm}^{-1}$ with opposite signs were observed. As clearly seen in the original spectra (panel (a)), both the clear peak and the two shoulders should be characteristic bands for ice. In the $T$ ranges of $C 3$ and $H 1$, water is in the solid state (ice). A symmetric band at $\sim 3450 \mathrm{~cm}^{-1}$ and two sharp bands with opposite signs at 3230 and $3150 \mathrm{~cm}^{-1}$ indicate the exchange from/to weakly H-bonded water molecules to/from strongly H-bonded ones by cooling/heating.

\section{B. Sorbed Water (Figures 3 and 4).}

Cooling Process. An increase in MIR absorption of sorbed water and a red-shift of the characteristic band of the bridging water at $\sim 3615 \mathrm{~cm}^{-1}$ were observed (panel (a)). If the red-shift is accompanied by the increase, the positive peak in the difference spectra (panel (b)) should be larger than the negative one. The difference spectrum of pBMA in the $T$ range of $C 1$ (Figure 3 ) is a typical case of this. However, in many cases, negative/positive peaks with comparable intensities or no positive peak were observed. These results clearly indicate that the sorbed water changes from the monomeric state to the associated one. In the cases of pEEMA (Figure 4) and pMA (Figure S1), the negative/positive peaks at $\sim 3650 / \sim 3600 \mathrm{~cm}^{-1}$ of the difference spectra in all $T$ ranges (panel (c)) have comparable intensities. Their spectral profiles in the low frequency region resemble neither the original spectrum of pure water (dotted line) nor that of ice. In addition, no drastic change in A3280 was observed (panel (b)). These changes suggest that most of the sorbed water has no change in the state such as condensation or crystallization and that a part of the sorbed water becomes a 
small water cluster by cooling.

In marked contrast to the above cases, drastic changes in A3280 and $\mathrm{A}_{\mathrm{MONO}}$ in the cases of pBMA (Figure 3) and pEEA (Figure S2) were observed. In the $T$ range of $C 2$, a broad absorption of the difference spectra (panel (b)) in the low frequency is similar to the original spectrum of pure water (dotted line). The values of A3280 and $\mathrm{A}_{\mathrm{MONO}}$ increase gradually and level off, respectively. The level-off in $\mathrm{A}_{\mathrm{MONO}}$ means a decrease of the bridging water. These results exactly exhibit the change of sorbed water from the monomeric state to liquid state. In the $T$ range of $C 3$, the similarity of difference spectra to the original spectra of ice (dotted line) in the low frequency region is evidence of the formation of ice. A steep but not discontinuous change in A3280 is different from the case of pure water. There are two possible explanations for this: one is that there is a freezing distribution depending on random fluctuation in the arrangement of nearby water molecules in the liquid state and another is that the bridging water is changed directly to ice without condensation. The latter process is denoted as "vapor deposition". The distribution of freezing point has been reported to be no more than $\sim 5 \mathrm{~K}^{38-41}$ strongly suggesting that this slow crystallization is caused mainly by vapor deposition. Two pieces of spectral evidence for this process are the decrease in $A_{\text {MONO }}$ synchronizing with the increase in A3280 and the absence of a positive peak in the high frequency region in the difference spectra. In the $T$ range of $C 4$ for pBMA, vapor deposition should be the sole process for the growth of ice because the difference spectrum is superposable on the original spectrum of ice (dotted line). In the lowest $T$ range for pBMA $(C 5)$ and pEEA $(C 4)$, the difference spectra in the low frequency region are almost the same as the spectrum of ice, which exhibits no more change in the state of the sorbed water in this $T$ range (although there is change in the state of ice already formed).

Heating Process. If recrystallization of the sorbed water occurs, the increase in the value of A3280 (or the decrease in the decreasing ratio of A3280) might be observed. Furthermore, if recrystallization is not devitrification but vapor re-deposition, the enhancement of the decrease in the value of $\mathrm{A}_{\mathrm{MONO}}$ should be observed at the same time.

In the lowest $T$ range $(H I)$, the spectral changes were a mirror image to those in the lowest $T$ range of the cooling process, indicating that the state change in the $T$ region is in the opposite direction of that in the cooling process. In the next $T$ range ( $H 2$ ) for pEEMA (Figure 4), pMA (Figure S1) and pEEA (Figure S2), the decrease in $\mathrm{A}_{\text {MONo was enhanced }}$ and the value of A3280 increased. In the two cases for pEEMA and pMA, although no ice formation during cooling was observed, a large negative band in the difference spectra in the $T$ range of $H 3$ is similar to the spectrum of ice in the opposite sign (dotted line). This clearly confirms the decrease in ice in the $T$ range of $H 3$ and the presence of ice at the low side of this $T$ range. The increase in A3280 in the $T$ range of $H 2$ for the three polymers, therefore, corresponds to the growth of ice. This is just recrystallization of the sorbed water. It is noted here that the increase in A3280 coincides with the enhancement of decrease in $\mathrm{A}_{\mathrm{MONO}}$. This suggests that recrystallization is a direct change from the bridging water to ice. This process is denoted as "vapor re-deposition". ${ }^{42}$

In the case of pBMA (Figure 3), the change in A3280 and the difference spectrum in the Trange of $H 2$ are different from the above three cases, although enhanced decrease in $A_{M O N O}$ is observed. An increase in A3280 was not observed, but the rate of decrease in A3280 became smaller. The value of A3280 for ice decreases linearly until melting (right of panel (b) in Figure 2). This suggests that the decrease in the rate of decrease in A3280 means growth of ice. Therefore, the change in A3280 observed in the case of pBMA also indicates growth of ice during heating. This growth of ice is also considered to be vapor re-deposition because the enhanced decrease in $\mathrm{A}_{\mathrm{MONO}}$ was obtained simultaneously.

As stated above, in the $T$ range of $H 3$, a large negative band being similar to the spectrum of ice in the difference spectra was observed in all cases. This clearly indicates decrease in ice in this $T$ range. It is noted here that the change in A3280 is different from that when ice melts (Figure 2): the change was very steep but not discontinuous. The very large positive peak attributed to the bridging water and the increase in $A_{\text {MONO }}$ synchronizing with the decrease in A3280 indicate that most of ice formed in the polymer matrix directly transforms to the bridging water. This process is denoted as "sublimation". However, in the cases of pBMA (Figure 3) and pMA (Figure S1), the difference spectrum had a very broad negative band that is superposable on the original spectrum of liquid water (dotted line) in the next $T$ range $(H 4)$. This suggests that a part of the decrease in ice in the $T$ range of $H 3$ in pMA and pBMA is melting and this liquid water is vaporized in the $T$ range of $H 4$. On the other hand, a liquid-like spectrum of water was not observed in the difference spectra of pEEMA (Figure 4) and pEEA (Figure S2) in any of the $T$ ranges, indicating that the decrease in ice in the two polymers should be only by sublimation.

\section{Discussion}

Change in the State of Sorbed Water All of water sorbed into the non-water soluble polymers by vapor sorption has been widely considered to be the non-freezing water. In other words, water molecules are maintained in the monomeric state at any $T$. This is mainly based on DSC analysis. No DSC signal from water sorbed into pBMA, ${ }^{15}$ poly(n-butyl acrylate) (pBA), ${ }^{20}$ and $\mathrm{pMEA}^{20}$ as the analogue of pEEA and pEEMA has been reported. However, in the first place, there are not many reports on sorbed water by vapor sorption on the basis of DSC analysis. This might be because many researchers (including our research group) have been under the impression that the sorbed water should not be frozen. Besides, MIR spectroscopic analysis of various hydrated poly(meth)acrylates ${ }^{19-22,43-46}$ at room $T$ has also abundantly reported the same interpretation. These spectral profiles of the sorbed water including the four polymers in this study (Figure 1) did not resemble that of pure water at all. The large difference hardly allows us to consider the 
change in the state by $T$. In our previous reports on several kinds of poly(meth)acrylates ${ }^{19,45}$ at room $T$, we also imprudently had the same interpretation without examining $T$ dependence of MIR spectra of the sorbed water.

As mentioned above, it should be correct that the sorbed water is in a monomeric state at room $T$. However, the present results and our recent studies on poly(methyl methacrylate) (pMMA), ${ }^{30} \mathrm{pMEA}^{29}{ }^{\mathrm{pBA}},{ }^{47}$ and polystyrene (PS) ${ }^{48}$ by $T$ variable MIR spectroscopy demonstrate that the change in the state by $T$ is an undeniable fact. That is to say, the fact that the sorbed water is in a monomeric state at room $T$ should not always mean that all of it is non-freezing water. Changes in the state observed spectroscopically, including changes observed in our recent studies, are summarized in Table 2. These changes should be observed as a DSC signal if DSC sensitivity is high enough to detect all of the heat flux in the system (although it might be difficult for the current apparatus generally used).

Heat flux with condensation/vaporization of the sorbed water, nevertheless, might be more difficult to detect by DSC without the relation to its sensitivity. This is because the vaporization/condensation enthalpy of pure water, \pm 44.9 $\mathrm{kJ} \cdot \mathrm{mol}^{-1}$ at $273 \mathrm{~K},{ }^{23}$ is comparable to the sorption/desorption ones of water molecules to/from the non-water soluble poly(meth)acrylates, $\sim 40 \mathrm{~kJ} \cdot \mathrm{mol}^{-1}$ at room $T$, in opposite signs. ${ }^{47}$ Condensation/vaporization of the sorbed water should be accompanied by desorption/adsorption of water molecules from/to the polymer chain, resulting in the two enthalpies canceling out each other. For the same reason, with sublimation/vapor deposition (including vapor re-deposition), the DSC signal expected from sublimation/vapor deposition enthalpy of pure water, $\pm 50.8 \mathrm{~kJ} \cdot \mathrm{mol}^{-1}$ at $273 \mathrm{~K},{ }^{23}$ should not be obtained but only that near to melting/freezing enthalpy of pure water, $\pm 6.0 \mathrm{~kJ} \cdot \mathrm{mol}^{-1}$. These phenomena have been demonstrated in the hydrated pBA system by high-sensitive DSC and $T$ variable MIR spectroscopy. ${ }^{47}$ These situations frequently lead to a completely misleading interpretation that liquid water (condensed water) is present at room $T$.

Various changes in the state given in Table 2 might hardly be accepted from the viewpoint of interpretation on the basis of DSC analysis. However, if accepting that the polymer chains are a part of the components of a small holed cage confining water molecules and that the polymer matrix is only an assembly of the cages, the phenomena observed here might not be strange. This is because the three states of matter (solid, liquid, and gas) depend only on the chemical potential: the matter has an association tendency with $T$ reduction. ${ }^{49}$ It is also clear, on the other hand, that the polymer chain sterically and physically (due to electrostatic interaction (H-bonding interaction)) affects the diffusion of water molecules and the association between water molecules. The degree of the effect should be dependent on the glass transition $T\left(T_{\mathrm{g}}\right)$ and the number of electrostatic interaction sites (oxygen atoms). ${ }^{50}$ The number of oxygen atoms per unit volume of polymers calculated from the density of polymers ${ }^{51-56}$ and its chemical formula is given in Table 2 . In general, the greater the number of H-bonding sites is, the more inhibited are the diffusion and the association. The greater the reduction in $T_{\mathrm{g}}$ 's is, on the other hand, the less inhibited is the diffusion.

Although exact estimation of change in the state is difficult, some tendencies can be obtained: (1) a polymer with high $T_{\mathrm{g}}$ and many H-bonding sites such as pMMA shows no change in the state; (2) a polymer with low $T_{\mathrm{g}}$ and few H-bonding sites such as pBA shows change in the state except for recrystallization (vapor re-deposition); (3) a polymer with high $T_{\mathrm{g}}$ and few H-bonding sites such as pBMA and PS shows all of the changes in the state including recrystallization (vapor re-deposition); and (4) a polymer with low $T_{\mathrm{g}}$ and many H-bonding sites such as pMEA, pMA and pEEMA shows no liquid state but crystallization. More discussion on recrystallization (vapor re-deposition) is presented below.

Recrystallization (Vapor Re-deposition) Recrystallization of water has been believed to be rare for non-water soluble polymer systems. There are some reports about recrystallization of water for a highly concentrated aqueous solution of polymers ${ }^{24-28}$ but, as far we know, there have been only two reports for non-water soluble polymer systems $\left(\right.$ pMEA $^{10}$ and pTHFA $\left.^{14}\right)$. Regardless of their water solubilities, recrystallization was considered to be devitrification, which is the change from glassy water to ice during heating. Devitrification, of course, is observed in the case of pure water, but a special technique such as a hyperquenching method is required. ${ }^{57-59}$ Recrystallization of water (maybe devitrification) observed in aqueous polymer solutions also generally requires rapid cooling. ${ }^{24-28}$ On the other hand, in the cases of pMEA and PTHFA, recrystallization was demonstrated by DSC even at a slow cooling rate of 2.5 $\mathrm{K} \cdot \mathrm{min}^{-1} \cdot{ }^{10,14}$ Furthermore, our recent study on $\mathrm{pMEA}^{29}$ and the present results for the four polymers demonstrated recrystallization at extremely slow cooling rates of 0.25 and $0.5 \mathrm{~K} \cdot \mathrm{min}^{-1}$, respectively. These phenomena are very interesting and worth special attention if it is true that recrystallization in their polymer matrices is devitrification. As demonstrated by the results, however, the $T$ dependence of MIR spectra clearly indicates that recrystallization is vapor re-deposition and not devitrification. This is also supported by the dissimilar spectra in all $T$ ranges to those of glassy water $^{60}$ and low-density liquid water ${ }^{61}$. Devitrification and vapor re-deposition are the same in the point that crystalline water is formed during heating, but the two phenomena are essentially different.

The recrystallization by vapor re-deposition might be related to the balance between flexibility of the polymer chain and strength of the electrostatic interaction with water molecules. Figure 5 shows a plot of starting $T$ 's of recrystallization of water, $T_{\mathrm{rc}}$ 's, (Table 2) against $T_{\mathrm{g}}$ 's of the polymers. In the cases of the polymers with high $O$ atom density and low $T_{\mathrm{g}}$ (pMA, pEEA, pEEMA and pMEA), the value of $T_{\mathrm{rc}}$ increased with increase in $T_{\mathrm{g}}$. This tendency could be explained as follows: the diffusion of the bridging water, which is restricted to the electrostatic interaction sites below $T_{\mathrm{g}}$ of polymers, is spuriously enhanced with the increase in flexibility of the polymer chains during heating (that is, glass-to-rubber transition of the polymer), resulting in enhancement of the association between water molecules, and 
then if $T$ at that moment is lower than $T_{\mathrm{m}}$ of water, the bridging water might be recrystallized (vapor re-deposited). The reason why $T_{\mathrm{rc}}$ 's are lower than $T_{\mathrm{g}}$ 's is that $T_{\mathrm{g}}$ 's are not defined as starting $T$ 's of glass-to-rubber transition, which should be lower than $T_{\mathrm{g}}$ 's. ${ }^{62}$

In the cases of polymers with relative high $T_{\mathrm{g}}$ and low $O$ atom density (pBMA) and without $O$ atom (PS), $T_{\text {rc }}$ 's decreased with increase in $T_{\mathrm{g}}$. In contrast to the above cases (pMA, pEEA, pEEMA and pMEA), this tendency might be related to only strength of the electrostatic interaction rather than flexibility of the polymer chain. This is because their $T_{\mathrm{g}}$ 's are higher than $T_{\mathrm{m}}$ of water by more than $20 \mathrm{~K}$ and therefore the polymer chain is considered to be completely in the glass state at their $T_{\text {rc }}$ 's. It might be better to consider that recrystallization (vapor re-deposition) in the two cases occurred when the diffusion force of water molecules exceeds the restriction force by the electrostatic interaction. That the diffusion force of any molecules increases with heating is the physicochemical requirement. The electrostatic interaction of $O$ atoms in (meth)acrylate polymers with water molecules should be much stronger than that of $\pi$-electrons of the phenyl ring in PS. ${ }^{63}$ This order does not conflict with the order of $T_{\mathrm{rc}}$ for them.

The consideration mentioned above means that the recrystallized water is merely the bridging water, which should be condensed and/or vapor-deposited though the diffusion during cooling and which might be retained at $T$ at which the water molecules could not diffuse due to a high cooling rate. It also means that the mass of the recrystallized water is decreased at slower cooling and is not observed at infinitely slow cooling because the sorbed water should be frozen during cooling. Strong dependence of the mass of the recrystallized water in pMEA and PS on cooling rate has been reported: the slower cooling rate diminished the mass of the recrystallized water.

\section{Conclusion}

After the two reports for recrystallization of water in non-water soluble polymers (pMEA and pTHFA) on the basis of DSC analysis, recrystallization was newly demonstrated spectroscopically in four poly(meth)acrylates in this study. The four cases clearly indicate that recrystallization is no longer a rare phenomenon. In addition, different from aqueous polymer solutions, all of the recrystallization observed in this study is not devitrification but vapor re-deposition. Needless to say, condensed water with a special structure to recrystallize in non-water soluble polymers does not exist at physiological $T$. Furthermore, recrystallization of water is not related to the biocompatibility of polymers. It rather depends on the balance between flexibility and strength of the electrostatic interaction of polymers with water molecules.

\section{Acknowledgments}

This research was supported by a Grant-in-Aid for Scientific Research (22350101) from the Japan Society for the Promotion of Science and a Grant-in-Aid for Scientific Research on Innovative Areas (20106007) from the Ministry of Education, Culture, Sports, Science and Technology, Japan.

Supporting Information Available: Temperature dependence of the MIR spectra for pMA and pEEA. This materials is available free of charge via the Internet at http://pubs.acs.org.

\section{References and Notes}

1) Okano, T.; Nishiyama, S.; Shinohara, I.; Akaike, T.; Sakurai, Y.; Kataoka, K.; Tsuruta, T. J. Biomed. Mater. Res. 1981, $15,393$.

2) Stevens, M. M. and Gerge, J. H. Science 2005, 310, 1135.

3) Discher, D. E.; Mooney, D. J. Zandstra, P. W. Science 2009, 324, 1673.

4) Mitragotri, S. and Lahann, J. Nature Mater. 2009, 8, 457.

5) Place, E. S.; Evans, N. D.; Stevens, M. M. Nature Mater. 2009, 8, 457.

6) Kitano, H. and Gemme-Ide, M. J. Biomater. Sci. Polym. Ed. 2010, 21, 45.

7) Kitano, H.; Sudo, K.; Ichikawa, K.; Ide, M.; Ishihara, K. J. Phys. Chem. B 2000, 104, 11425.

8) Ishihara, K.; Nomura, H.; Mihara, T.; Kurita, K.; Iwasaki, Y.; Nakabayashi, N. J. Biomed. Mater. Res. $1998,39,323$.

9) Goda, T.; Watanabe, J.; Takai, M.; Ishihara, K. Polymer 2006, 47, 1390.

10) Tanaka, M.; Motomura, T.; Ishii, N.; Shimura, K.; Onishi, M.; Mochizuki, A.; Hatakeyama, T. Polym. Int. 2000, 49, 1709. The value of $T_{\mathrm{g}}$ for pMEA with $M_{\mathrm{w}}=85,000$ was determined by DSC.

11) Tanaka, M.; Mochizuki, A.; Ishii, N.; Motomura, T.; Hatakeyama, T. Biomacromolecules 2002, 3, 36.

12) Tanaka, M. and Mochizuki, A. J. Biomed. Mater. Res. A 2004, 68A, 684.

13) Hirota, E.; Tanaka, M.; Mochizuki, A. J. Biomed. Mater. Res. A 2007, 81A, 710.

14) Mochizuki, A.; Hatakeyama, T.; Tomono, Y.; Tanaka, M. J. Biomater. Sci. Polym. Ed. 2009, $20,591$.

15) Mochizuki, A.; Kimura, M.; Ina, A.; Tomono, Y.; Tanaka, M. J. Biomater. Sci. Polym. Ed. 2010, $21,1895$.

16) Tanaka, M. and Mochizuki, A. J. Biomater. Sci. Polym. Ed. 2010, 21, 1849.

17) Kishi, A.; Tanaka, M.; Mochizuki, A. J. App. Polym. Sci. 2009, 111, 476.

18) Miwa, Y.; Ishida, H.; Tanaka, M.; Mochizuki, A. J. Biomater. Sci. Polym. Ed. 2010, $21,1911$.

19) Ide, M.; Mori, T.; Ichikawa, K.; Kitano, H.; Tanaka, M.; Mochizuki, A.; Oshiyama, H.; Mizuno, W. Langmuir 2003, 
19,429 .

20) Morita, S.; Tanaka, M.; Ozaki, Y. Langmuir 2007, 23, 3750.

21) Tanabe, A.; Morita, S.; Tanaka, M.; Ozaki, Y. App. Spectroscopy 2008, 62, 46.

22) Morita, S.; Tanaka, M.; Kitagawa, K.; Ozaki, Y. J. Biomater. Sci. Polym. Ed. 2010, 21, 1925.

23) Washbum, E. W. In International Critical Tables of Numerical Data, Physics, Chemistry and Technology; McGraw-Hill: New York, 1929; Vol. 5, pp131-138.

24) Franks, F. In Water: A Comprehensive Treatise; Franks, F.; Ed.; Plenum Press: New York, 1982; Vol. 7, pp215-338.

25) Graham, N. B.; Zulfiqar, M.; Nwachuku, N. E.; Rashid, A. Polymer 1989, 30, 528.

26) Hang, L. and Nishinari, K. J. Polym. Sci. B: Polym. Phys. 2001, 39, 496.

27) Hatakeyama, T.; Tanaka, M.; Hatakeya, H. J. Biomaterials Sci. 2010, 21, 1865.

28) Zhang, Z. L. and Ling, G. N. Physiol. Chem. Med. NMR 1983, 15, 407.

29) Gemmei-Ide, M. and Kitano, H. J. Phys. Chem. B 2008, 112, 12863.

30) Gemmei-Ide, M.; Motonaga, T.; Kitano, H. J. Phys. Chem. B 2007, 111, 5533.

31) Scherer, J. R. In Advances in Infrared and Raman Spectroscopy, edited Clark, R. J. H. and Hester, R. E., Heyden: Philadelphia, 1978; Vol. 5, p. 149.

32) Du, Q.; Superfine, R.; Freysz, E, Shen, Y. R. Phys. Rev. Lett. 1993, 70, 2313.

33) Iwamoto, R. J. Phys. Chem. A 2010, 114, 7398.

34) Iwamoto, R.; Matsuda, T.; Sasaki, T.; Kusanagi, H. J. Phys. Chem. B 2003, 107, 7976.

35) Eisenberg, D.; Kauzmann, W.; In The Structure and Properties of Water; Clarendon Press: London, 1696; Chapter 4.

36) Walrafen, G. E.; Chu, Y. C. J. Phys. Chem. 1995, 99, 11225.

37) Ohno, K.; Okimura, M.; Akai, N.; Katsumoto, Y. Phys. Chem. Chem. Phys. 2005, 7, 3005.

38) Cwilong, B. M. Proc. Roy. Soc. London 1947, A190, 137.

39) Schaefer V. J. Ind. Eng. Chem. 1950, 44, 1300.

40) Bigg, E. K. Quart. L. Met. Soc. 1953, 79, 510.

41) Clausse, D.; Dumas, J. P.; Broto, F. C. R. Acad. Sci. Paris 1974, 279B, 415.

42) The term "vapor re-deposition" means vapor deposition during heating and is an imitation of the term "recrystallization". This is to avoid confusion with the term "vapor redeposition" frequently used in the field of solid surface processing via vapor deposition.

43) Sutandar, P.; Ahn, D. J.; Franses, E. I. Macromolecules, 1994, 27, 7316.

44) Kusanagi, H.; Yukawa, S. Polymer 1994, 35, 5637.

45) Ichikawa, K.; Mori, T.; Kitano, H.; Fukuda, M.; Mochizuki, A.; Tanaka, M. J. Polym. Sci. B Polym. Phys. 2001, 39, 2175.

46) Shephard, J. J.; Bremer, P. J.; McQuillan, A. J. J. Phys. Chem. B 2009, 113, 14229.

47) Gemmei-Ide, M.; Ohya, A.; Kitano, H. J. Phys. Chem. B 2010, 114, 4310.

48) Gemmei-Ide, M.; Kitano H. J. Phys. Chem. B 2008, 112, 13499.

49) McQuarrie, D.; Simon, J. In Physical Chemistry: A Molecular Approach; University Science Books: Sansalito, California, 1997.

50) Takizawa, A.; Hamada, T.; Okada, H.; Kadota, S. Polymer 1974, 15, 157.

51) Cowie, J. M. G.; Reilly, A. A. N.; J. App. Polym. Sci. 1993, 47, 1155. The value of $T_{\mathrm{g}}$ for pMA with $M_{\mathrm{n}}=57,000$ was determined by DSC.

52) Nikitin, L. N.; Said-Galiyev, E. E.; Vinokur, R. A.; Khokhlov, A. R.; Gallyamov, M. O.; Schaumburg, K. Macromolecules, 2002, 35, 934. The values of $T_{\mathrm{g}}$ for pMMA with $M_{\mathrm{w}}=120,000$ and for pBMA with $M_{\mathrm{w}}=320,000$ were determined by DSC.

53) DTMA, Mw no, Tiemblo, P.; Laguna, M. F.; Garcia, F.; Garcia, J. M.; Riande, E.; Guzman, J. Macromolecules 2004, 37, 4156. The value of $T_{\mathrm{g}}$ for pEEMA was determined by dynamic thermomechanical analysis. pEEMA used in this literature has a very low cross-link density (vinyl monomer : cross-linking agent $=600: 1$ ).

54) Kano, Y.; Akiyama, S.; Fujita, Y. Polym. Networks Blends 1996, 6, 105. The value of $T_{\mathrm{g}}$ for $\mathrm{pBA}$ with $M_{\mathrm{w}}=142,000$ was determined by DSC.

55) Guschl, P. and Otaigbe, J. U. J. Colloid Interface Sci. 2003, 266, 82. The value of $T_{\mathrm{g}}$ for PS with $M_{\mathrm{w}}=230,000$ was determined by DSC.

56) Bovey, F. A.; Abere, J. F. , J. Polym. Sci. 1955, 15, 537. The value of $T_{\mathrm{g}}$ for pEEA was determined by refractometer. The value of $M_{\mathrm{w}}$ was not given.

57) Brüeggeller, P.; Mayer, E. Nature 1980, 288B, 569.

58) Brüeggeller, P.; Mayer, E. Nature 1982, 298, 715.

59) Kim, W.-H.; McPhillen, M.; Hayes, J. M.; Small, G. J. Chem. Phys. Lett. 1983, 207, 443.

60) Given, A.; Loewenshuss, A.; Nielsen, C. J. J. Phys. Chem. B 1997, 101, 8696.

61) Mallamace, F.; Broccio, M.; Corsaro, C.; Faraone, A.; Majolino, D.; Venuti, V.; Liu, L.; Mou, C. Y.; Chen, S. H. Proc. Natl. Acad. Sci. U.S.A. 2005, 104, 424.

62) Nakamura, S.; Todoki, M.; Nakamura, K.; Kanetsuna, H. Thermochim. Acta 1988, 136, 163. 
63) Gruenloh, C. J.; Carney, J. R.; Arrington, C. A.; Zwier, T. S.; Fredericks, S. Y.; Jordan, K. D. Science 1997, 276, 1678 .

Table 1. Characteristics of Polymers Used.

\begin{tabular}{|c|c|c|c|c|c|c|c|c|}
\hline Polymer & pMA $^{*}$ & pBMA $^{*}$ & pEEA $^{*}$ & pEEMA $^{*}$ & $\mathrm{pMMA}^{\mathrm{a}}$ & $\mathrm{pBA}^{\mathrm{b}}$ & pMEA $^{\mathrm{c}}$ & $\mathrm{PS}^{\mathrm{d}}$ \\
\hline$M_{\mathrm{w}} \times 10^{3}\left(M_{\mathrm{w}} / M_{\mathrm{n}}\right)$ & 77 (1.9) & $54(1.8)$ & $75(4.8)$ & $61(4.4)$ & $900(1.4)$ & $79(1.5)$ & $86(1.7)$ & $900(1.4)$ \\
\hline Water content / wt\% & 1.83 & 0.20 & 2.42 & 1.23 & 5.48 & 0.44 & 3.20 & 0.026 \\
\hline$T_{\mathrm{g}} / \mathrm{K}$ & $280^{\mathrm{e}}$ & $288^{\mathrm{f}}$ & $223^{g}$ & $257^{\mathrm{h}}$ & $412^{\mathrm{f}}$ & $219^{\mathrm{i}}$ & $223^{j}$ & $373^{\mathrm{k}}$ \\
\hline Density $/ \mathrm{g} \cdot \mathrm{cm}^{-3}$ & $1.21^{\mathrm{e}}$ & $1.07^{\mathrm{f}}$ & $(1.11)^{\dagger}$ & $1.11^{\mathrm{h}}$ & $1.19^{\mathrm{f}}$ & $1.06^{\mathrm{i}}$ & $(1.11)^{\dagger}$ & $1.04^{\mathrm{k}}$ \\
\hline $\begin{array}{l}\text { Number of } \mathrm{O} \text { atoms / } \\
\mathrm{mol} \cdot \mathrm{cm}^{-3}\end{array}$ & 0.0281 & 0.0148 & 0.0231 & 0.0211 & 0.0236 & 0.0165 & 0.0256 & - \\
\hline
\end{tabular}

Table 2. Change in the State of the Sorbed Water Observed Spectroscopically.

\begin{tabular}{|c|c|c|c|c|c|c|c|c|}
\hline Polymer & $\mathrm{pMA}^{*}$ & pBMA $^{*}$ & pEEA $^{*}$ & pEEMA $^{*}$ & pMMA $^{\mathrm{a}}$ & $\mathrm{pBA}^{\mathrm{b}}$ & pMEA $^{\mathrm{c}}$ & $\mathrm{PS}^{\mathrm{d}}$ \\
\hline \multicolumn{9}{|c|}{ Cooling process } \\
\hline Condensation & - & Yes & Yes & - & - & Yes & - & Yes \\
\hline Freezing & - & Yes & Yes & - & - & Yes & - & Yes \\
\hline Vapor deposition & - & Yes & Yes & - & - & Yes & Yes & Yes \\
\hline \multicolumn{9}{|c|}{ Heating process } \\
\hline $\begin{array}{l}\text { Vapor re-deposition } \\
\text { (Recrystallization) }\end{array}$ & Yes & Yes & Yes & Yes & - & - & Yes & Yes \\
\hline Sublimation & Yes & Yes & Yes & Yes & - & Yes & Yes & Yes \\
\hline Melting & - & Yes & - & - & - & Yes & - & Yes \\
\hline Vaporization & - & Yes & - & - & - & Yes & - & Yes \\
\hline$T_{\mathrm{rc}} / \mathrm{K}$ & 240 & 200 & 215 & 226 & - & - & 218 & 180 \\
\hline
\end{tabular}

*This work, ${ }^{\text {a }}$ Reference $30,{ }^{b}$ Reference $47,{ }^{c}$ Reference 48 and ${ }^{\mathrm{d}}$ Reference 29. 


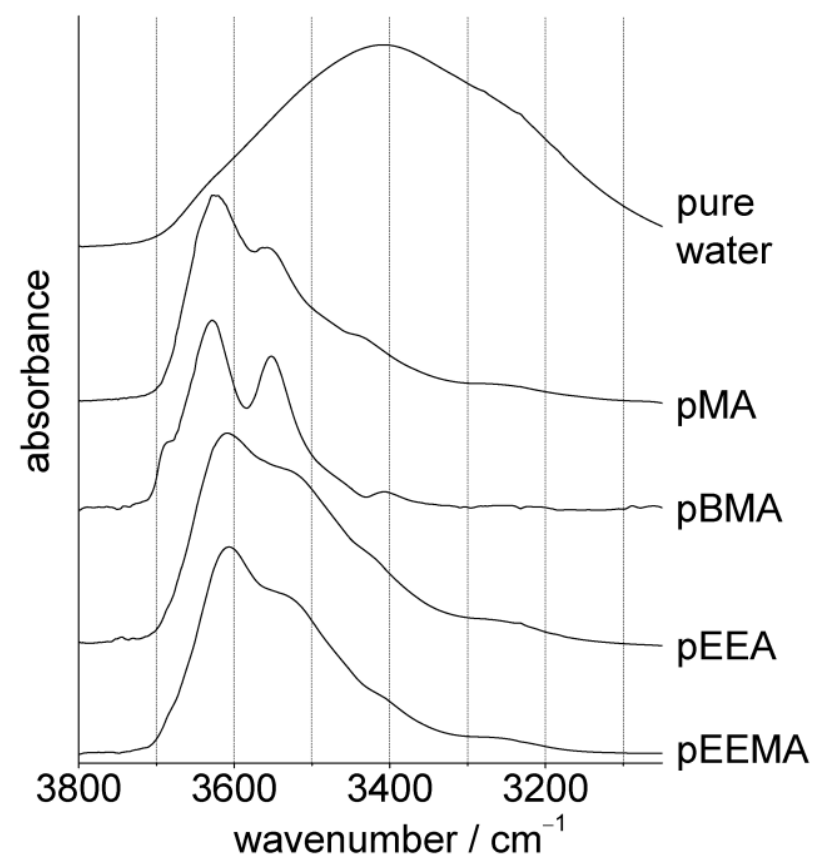

Figure 1. Gemmei-Ide et al. 9 

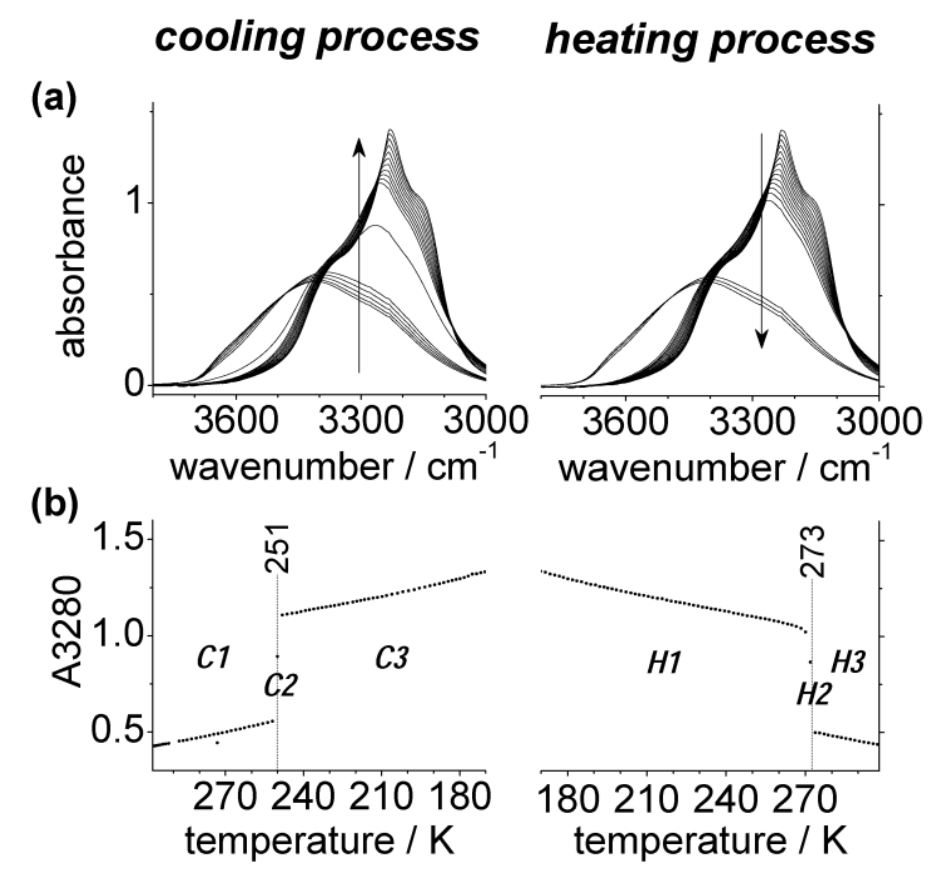

(c)

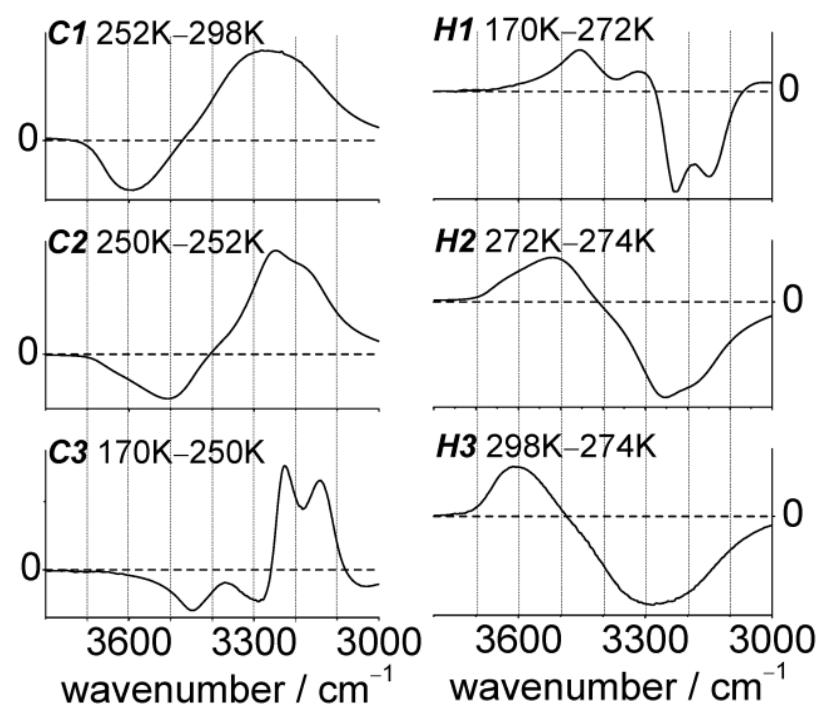

Figure 2. Gemmei-Ide et al10 


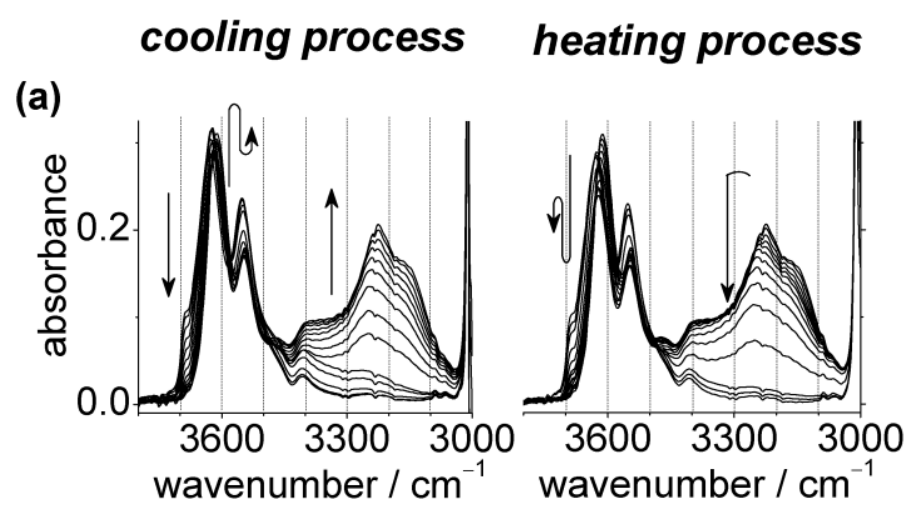

(b)


(c)
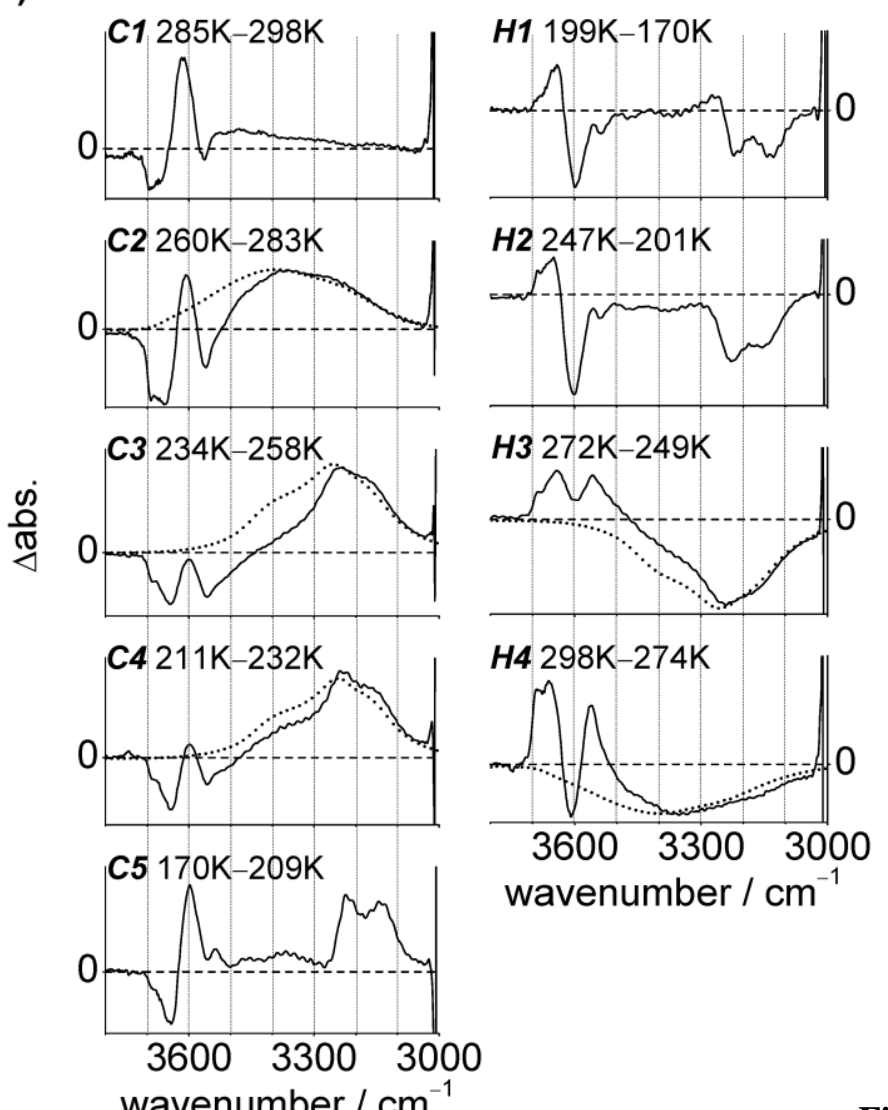

Figure 3. Gemmei-Ide et al. 
cooling process heating process
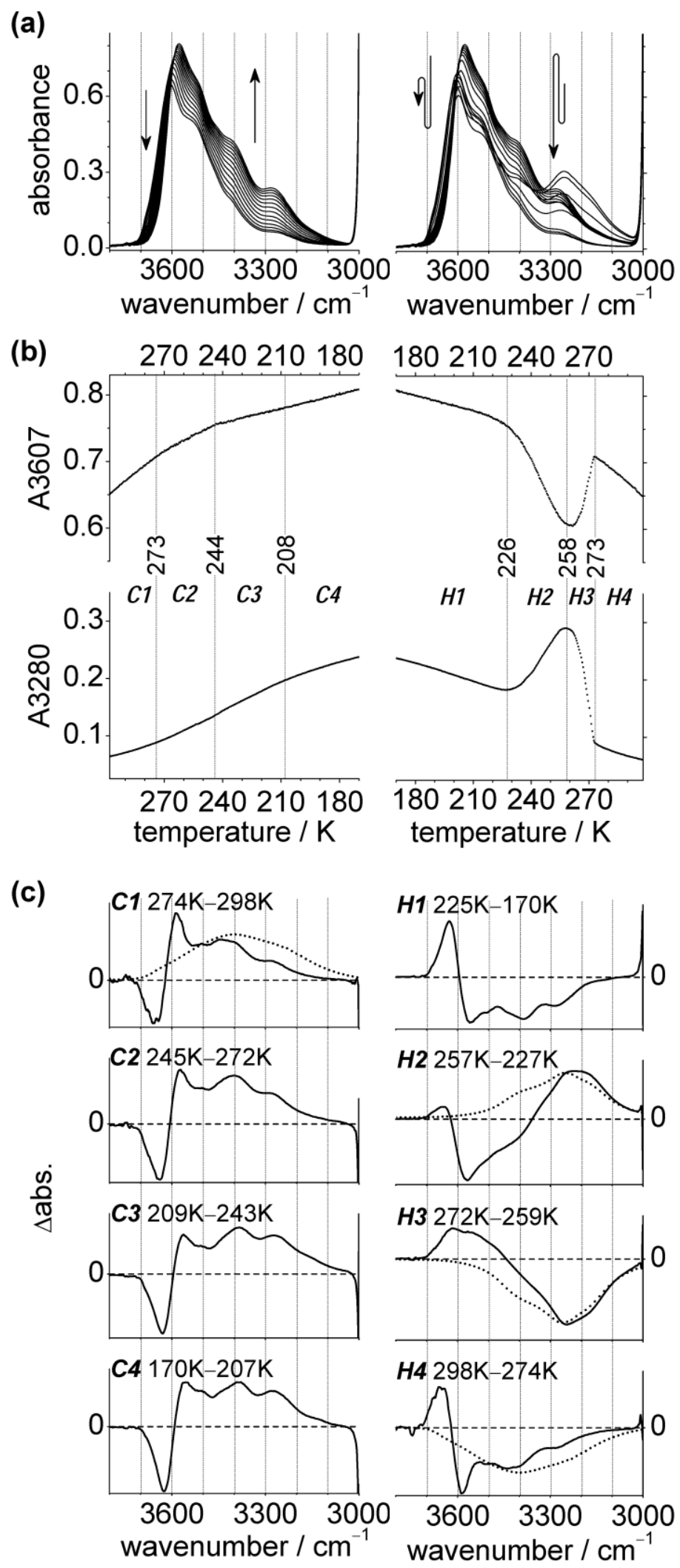

Figure 4. Gemmei-Ide et al. 




Figure 5. Gemmei-Ide et al. 
TOC



